\title{
Tailings storage facility surface moisture mapping for prediction of dusting events - and other benefits
}

\author{
J Thompson Fortescue Metals Group Ltd, Australia \\ L McDonald Fortescue Metals Group Ltd, Australia \\ J Kelcey NGIS Australia, Australia \\ J Dixon Fortescue Metals Group Ltd, Australia
}

\begin{abstract}
The fine-grained nature of some tailings materials can lead to dusting on tailings storage facilities (TSFs). Dusting has the potential to negatively affect the health of workers, nearby communities, and the surrounding environment which may adversely affect a mining operator's ability to operate. Mitigation measures to prevent dusting can be expensive and aren't always successful. Advances in monitoring systems allow the relative surface moisture of tailings beaches to be monitored during operations providing the opportunity to identify and forward plan the depositional strategy to minimise the risk of dusting. This paper presents the outcomes of a study that utilised predictive methods based on advanced synthetic aperture radar and multispectral data coupled with Google Earth Engine to develop a model for particular TSFs based on historical records, observations, and material types.

Google Earth Engine brings the first opportunity to use a systematic and comprehensive combination of radar and visual-infrared satellite data. It was found that the synergy between the two data types could be used to offset the individual ambiguities of each, and the resulting method delivered a predictive dryness probability map and visual moisture/water depth/presence indicators that were able to be verified and made operational almost immediately.

On-ground visual records and aerial imagery provided qualitative verification of the approach. The methodology allows TSF operators a free, open source platform with which to monitor and map surface moisture, enabling proactive deposition decision-making to mitigate the risk of tailings dusting. Additional benefits realised include increased data on beach formation, channel and pond location (extent and to some degree depth), improving the accuracy of the TSF water balance. For the particular TSFs studied, the water balance is a critical control from a safety perspective to mitigate potential failure mechanisms, and from an operational perspective to maximise tailings density and water return to the plant.
\end{abstract}

Keywords: tailings storage facility, tailings, dusting, environment, monitoring, satellite, water balance

\section{Introduction}

Whilst not classed as a failure in the traditional sense, dusting is nonetheless a failure of the tailings storage facility (TSF) to fulfil its primary objective - the containment of tailings. Dusting occurs when fine-grained tailings with low to no cohesive properties dries to a point whereby it can mobilise under aeolian action. This failure of containment can have significant effects on the environment, local communities, the health of workers, as well as economic, legal and reputational damage to mining companies often all happening at once. In the wake of high profile TSF failures and the implementation of the Global Industry Standard on Tailings Management, relations between miners and community have never been more at the forefront.

Dust suppression in mining is mostly centred around reducing traffic and stockpile lift-off, with the use of watercarts and sprinkler systems as the main tools to control dusting. Tailings dams are generally not located in high traffic areas for mining activities, rendering them and the dust they can produce unseen and 
forgotten, generally only coming to light when the health of workers, environment or the community is adversely affected. Additionally, the logistics of keeping large areas in a moist condition to prevent lift-off whilst simultaneously not adding to the TSF water balance (and thus increasing the risk of failure) can be challenging.

In 2019, Western Australia accounted for $37 \%$ of the world's iron ore supply, amounting to $837 \mathrm{Mt}$ (Department of Jobs, Tourism, Science and Innovation 2020). The majority of this ore was mined in the Pilbara region where Fortescue Metals Group operates; an area with an arid and tropical climate which experiences high temperatures and irregular cyclonic rainfalls. The arid nature of this region creates the ideal environment for the drying and potential mobilisation of tailings that is susceptible to lift-off, and makes for a water-poor region with little to spare for excess dust suppression.

To aid in the mitigation of any potential dusting, a tool was developed that enables a TSF to be monitored, regardless of accessibility, so that the point at which tailings becomes very dry can be used to indicated when tailings is susceptible to dusting.

\subsection{Project sites, pilot site and tailings}

\subsubsection{TSFA2}

A mine site was identified for development in a particularly water-poor area of the Pilbara region leaving little in the way for controlling dust suppression of the planned TSF. The ore mined at this location is expected to produce over $530 \mathrm{Mt}$ of tailings during the 20 year life-of-mine. This large volume of tailings requires an equally large impoundment. A natural valley was chosen as the location with earthen/waste rock embankment dams planned to impound the valley. During the final stage, the impoundment area measures $2.5 \mathrm{~km}$ in width and $4.5 \mathrm{~km}$ in length, approximately $13.5 \mathrm{~km}^{2}$, and contains a number of inaccessible and hidden valleys, and low-lying zones. The natural topography of the impoundment means little can be done in the traditional sense of visual monitoring of tailings flow paths, beach development, pond formation, and importantly, monitoring for areas that are drying sufficiently to allow dusting. Whilst drone technology offers the ability to do this, it can be difficult to gauge from photographs the extent to which tailings has dried, leaving the analysis subjective.

To conserve water, options were considered beyond the scope of sprinkler systems and addition of extra water to the TSF site to control dusting. The operational philosophy proposed is to utilise the water already in the tailings slurry by rotation of spigots to catch areas of potential dusting; a potential limitation is being able to identify these areas ahead of time given the aforementioned topographical constraints.

\subsubsection{Pilot sites and tailings}

Prior to development of the mine, a small-scale pilot plant and operations were established along with a TSF; TSFA1. TSFA1, also a valley dam, was operational from 2014 to 2017 and contains approximately $0.5 \mathrm{Mt}$ of tailings. Particle size distribution testing of tailings produced at the pilot plant showed a $\mathrm{P}_{80}$ range between 42 to $75 \mu \mathrm{m}$, consistent with the properties of sediments that are susceptible to lift-off as described by Kok et al. (2012). Seasonal wetting of tailings at TSFA1 provided the opportune test site for monitoring drying and the point at which tailings may dust.

A number of other sites with good visual access and active tailings deposition were also identified for additional verification of the active wetting and drying of tailings; TSFB1 and TSFB2. The tailings produced at these locations, however, were not susceptible to dusting and, therefore, are not able to verify the point of dusting. They can, however, be used to visually observe and 'calibrate' wet, drying, and very dry areas whilst TSFA1 was dry and decommissioned. 


\subsection{Proposed solution}

Remote sensing (RS) is the science of acquiring and analysing information about a target object or geographic area without having direct physical contact. The most common technique in RS is to record electromagnetic radiation (EM) observations. EM covers a broad spectrum of wavelengths from visible light (blue, green, and red) through to infrared, microwave and radio waves (Table 1). EM may interact with surfaces in a number of ways, including reflection, scattering, and absorption. EM RS can be passive, assessing naturally emitted $E M$, or active, whereby reflection of emitted EM is analysed. The core concept of RS is that properties can be derived from a material based upon how EM interacts with it (Lillesand et al. 2015).

Table 1 Common portions of the electromagnetic spectrum and their corresponding wavelength range

\begin{tabular}{ll}
\hline Electromagnetic spectrum & Wavelength \\
\hline Ultraviolet & $300-400 \mathrm{~nm}$ \\
Blue & $400-500 \mathrm{~nm}$ \\
Green & $500-600 \mathrm{~nm}$ \\
Red & $600-700 \mathrm{~nm}$ \\
Near infrared (NIR) & $700-1,400 \mathrm{~nm}$ \\
Shortwave infrared (SWIR) & $1,400-3,000 \mathrm{~nm}$ \\
Mid-wave infrared (MIR) & $3,000-8,000 \mathrm{~nm}$ \\
Long-wave infrared (LIR) & $8,000-15,000 \mathrm{~nm}$ \\
Thermal infrared (TIR) & $900-14,000 \mathrm{~nm}$ \\
Microwave or radar & $0.1-100 \mathrm{~cm}$ \\
Radio & $>100 \mathrm{~cm}$ \\
\hline
\end{tabular}

\subsubsection{Active (SAR) water detection}

Synthetic aperture radar (SAR) is an active RS methodology in which the sensor emits a radar pulse, which typically reflects, scatters, or is absorbed when it strikes the ground surface. Rough surfaces are responsible for scattering, whereby an EM pulse is diffusely reflected in all directions. Conversely, smooth surfaces are responsible for specular reflection and reflect the incoming EM pulse at the same angle of incidence (Lillesand et al. 2015). A radar pulse can be absorbed by very dry substrates (Bousbih et al. 2018). This occurs when the pulse penetrates into the dry material, within which it undergoes multiple interactions and is lost. Figure 1 demonstrates three common interactions of a radar pulse striking the ground surface.

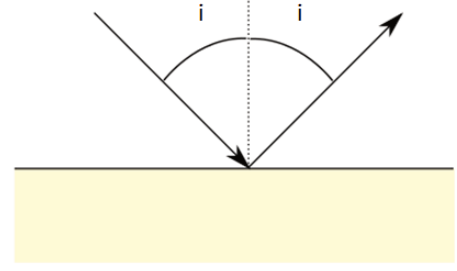

Reflection

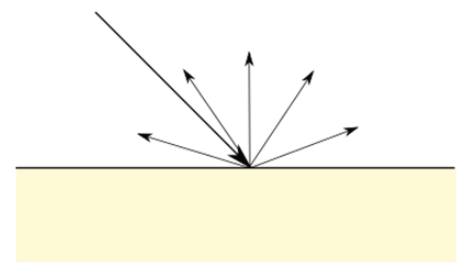

Scattering

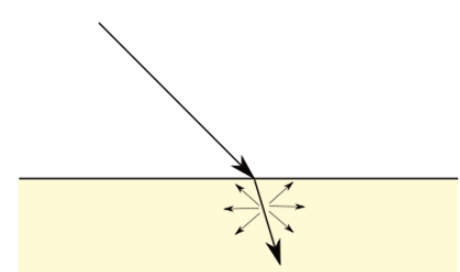

Absorption

Figure 1 Illustration of three common interactions of radar to a surface

In general, the EM scattering that returns back to the sensor (backscatter) is recorded as an observation, while the EM that reflects off the surface is lost. Through observations of backscattering, SAR can measure both the distance to the ground as well as its general structure. Distance is derived by measuring the direction 
and time elapsed between the emittance of the EM pulse and the recording of the backscatter (Lillesand et al. 2015). Measuring structure through SAR data is more complicated and is derived from combination of backscatter and polarisation. Interpretation of SAR data requires a highly skilled expert as similar raw data signals may have different causes depending on the physical context.

SAR backscatter is recorded as the ratio between the energy received and the energy emitted ( $d B)$. In addition, the energy that SAR transmits and receives may also be polarised by the sensor (Lillesand et al. 2015). The mode of polarisation of SAR data may either be vertical (V) or horizontal (H). An illustration of SAR polarisation is presented in Figure 2. As the target surface itself may also cause polarisation of the EM pulse, comparing different polarisation modes of the SAR sensor can be used to identify different structures.

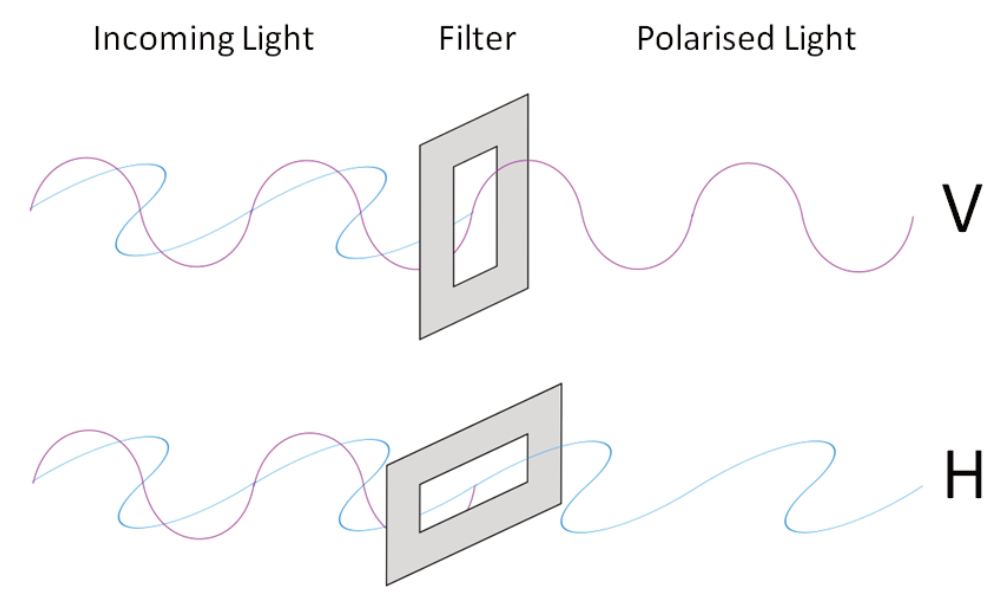

Figure 2 Illustration and comparison between $\mathrm{V}$ and $\mathrm{H}$ polarisation modes

The capacity for SAR to detect surface water has been widely demonstrated (Karvonen et al. 2005; Liang \& Liu 2020; Martinis et al. 2015). Surface water is capable of behaving as both a smooth surface and a rough surface. Still water typically acts as a specular reflector, which results in very low backscatter signals, while moving water can result in a high backscatter response due to a range of effects that include Bragg resonance (Lillesand et al. 2015). Care is still required to interpret low backscatter observations as they may also be the result of very dry substrates, i.e. it is difficult to differentiate accurately, through SAR backscatter alone, between water and very dry substrates.

\subsubsection{Passive (infrared) water detection}

A multispectral sensor passively records EM observations within discrete wavelength ranges (Lillesand et al. 2015). Typical ranges include visible light (blue, green, and red), the near infrared, and the shortwave infrared. The distance that EM can pass through the water column before it is completely absorbed is dependent upon its wavelength (Tian \& Philpot 2005). Water has a very strong absorbance signature in the infrared wavelength, absorbing almost all the light within this range. Thus, sensors using these wavelengths are very effective for identifying water. However, within these wavelengths, dry or very dry surfaces will still remain difficult to differentiate.

\section{$2 \quad$ Methodology}

\subsection{Study goal}

We can determine with some certainty from shortwave infrared (SWIR) data which surface areas are covered in water, and which are not. Similarly, we can determine from SAR data which surfaces are dry, as opposed to water covered or very dry. Thus, through data fusion, we aim to differentiate between all three surface states: water, dry, and very dry. 


\section{$2.2 \quad$ Study methodology}

\subsubsection{Very dry material detection}

SAR data representing three distinct and known surfaces was analysed: water, dry surface and very dry surface. The relationship between backscatter response and SWIR reflectance of these three classes is presented in Figure 3. These three classes form notable clusters along these response axes. Two features of this relationship are of note. Firstly, as discussed previously, SAR backscatter alone is relatively capable of separating water and dry material, but signals from very dry areas can also appear as similar to water covered areas. Secondly, SWIR data can allow adequate differentiation between those surfaces that are water covered and those that are not, but struggles to separate dry from very dry. These similarities and the relative strengths and weaknesses of each RS methodology are distinguishable in Figure 3. Based on this analysis of known data, we aim to develop a methodology utilising the properties of radar to separate dry from very dry materials, and the properties of SWIR to separate water from very dry, resulting in accurate identification of each surface type in out of sample data.

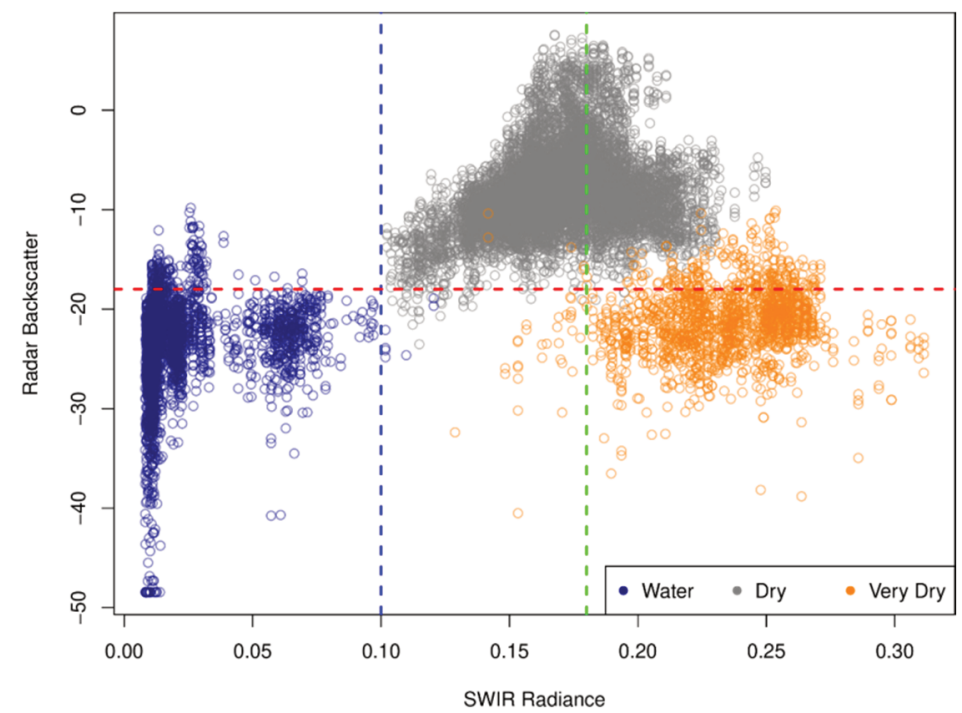

Figure 3 Scatter plot illustrating the relationship between radar and SWIR for three sampled TSF landcover classes: water, dry, and very dry

The distribution of very dry material was mapped using probabilistic modelling. Per-pixel probabilities were calculated on a gradient between water and very dry material. This was achieved by defining the statistical structure of both the water and very dry material clusters by sampling verified locations of known dryness and water (Table 2). The probability of membership to very dry material was estimated on a per-pixel basis using a maximum likelihood classifier.

Table 2 Statistical structure of water and very dry clusters relative to SAR backscatter and SWIR

\begin{tabular}{lllll}
\hline Cluster & \multicolumn{2}{c}{ VV backscatter $(\mathrm{dB})$} & \multicolumn{2}{c}{ SWIR (radiance) } \\
& Mean $(\boldsymbol{\mu})$ & Standard deviation $(\boldsymbol{\sigma})$ & Mean $(\boldsymbol{\mu})$ & Standard deviation $(\boldsymbol{\sigma})$ \\
\hline Water (W) & -23.78 & 4.79 & 219.24 & 190.67 \\
Very dry (VD) & -21.25 & 3.5 & $2,345.84$ & 259.51 \\
\hline
\end{tabular}

The maximum likelihood classifier is based on the standardised distance of per-pixel spectral values to those of the water and very dry clusters. In effect, the probability is the normalised distance from the water cluster (whereby 0 is within the water cluster and 1 would be within the very dry cluster). The probability of a given pixel belonging to the very dry cluster is calculated with Equation 1 . The normalised distance from the water cluster and from the very dry cluster is calculated using Equations 2 and 3, respectively. 


$$
P_{v . d r y}=\frac{\delta W}{\delta W+\delta V D}
$$

where:

$P_{V \cdot d r y}=$ the probability that a given pixel belongs to the very dry cluster.

$\delta W=$ the normalised distance from a pixel to the water cluster.

$\delta V D=$ the normalised distance from a pixel to the very dry cluster.

$$
\delta W=\sqrt{\left[\frac{S W I R-\left(S W I R \mu_{W}\right)}{\left(S W I R \sigma_{W}\right)}\right]^{2}+\left[\frac{V V-\left(V V \mu_{W}\right)}{\left(V V \sigma_{W}\right)}\right]^{2}}
$$

where:

$S W I R=$ the observed pixel SWIR radiance.

$S W I R_{\mu W}=$ the observed SWIR radiance mean of the water cluster.

$S W I R_{\sigma W}=$ the observed SWIR radiance standard deviation of the water cluster.

$V V=$ the observed pixel SAR VV backscatter.

$V V_{\mu W}=$ the observed SAR VV backscatter mean of the water cluster.

$V V_{\sigma W}=$ the observed SAR VV backscatter standard deviation of the water cluster.

$$
\delta V D=\sqrt{\left[\frac{S W I R-\left(S W I R \mu_{V D}\right)}{\left(S W I R \sigma_{V D}\right)}\right]^{2}+\left[\frac{V V-\left(V V \mu_{V D}\right)}{\left(V V \sigma_{V D}\right)}\right]^{2}}
$$

where:

$$
\begin{aligned}
& S W I R_{\mu V D}=\text { the observed SWIR radiance mean of the very dry cluster. } \\
& \text { SWIR }_{\sigma V D}=\text { the observed SWIR radiance standard deviation of the very dry cluster. } \\
& V V_{\mu V D}=\text { the observed SAR VV backscatter mean of the very dry cluster. } \\
& V V_{\sigma V D}=\text { the observed SAR VV backscatter standard deviation of the very dry cluster. }
\end{aligned}
$$

\section{Data}

Google Earth Engine (GEE) is a cloud infrastructure developed by Google for the large-scale analysis of geospatial data. GEE combines a multi-petabyte catalogue of satellite imagery and geospatial datasets with the capacity for planetary-scale RS analysis. This is achieved through an architecture built on parallelisation and horizontal scaling. The public data archives within GEE covers more than 30 years of historical imagery and includes the entire catalogue of both NASA's Landsat and the European Space Agency's Sentinel 1 and 2 programs.

\subsection{Sentinel 1}

Sentinel 1 is an imaging radar mission utilising SAR for broad-scale mapping of land and marine environments. The mission consists of a constellation of two satellites: Sentinel 1A and Sentinel 1B (Table 3). Interferometric wide (IW) swath is the primary mode of data collection over non-polar regions. Sentinel 1 collects both co-polarised and cross-polarised backscatter data. 
Table 3 Characteristics of the Sentinel 1 satellites

\begin{tabular}{llll}
\hline Commission & Spatial (metres) & $\begin{array}{l}\text { Revisit } \\
\text { (days) }\end{array}$ & Polarisation modes \\
\hline $2014(1 \mathrm{~A})$ & Stripmap: 84.0 & $1.5-4$ & Single $(\mathrm{VV}, \mathrm{HH})$ \\
$2016(1 \mathrm{~B})$ & $\begin{array}{l}\text { Interferometric wide swath: } 88.0 \times 87.0 \\
\end{array}$ & & Dual $(\mathrm{HH}+\mathrm{HV}, \mathrm{VV}+\mathrm{VH})$ \\
& $\begin{array}{l}\text { Extra wide swath: } 93.0 \times 87.0 \\
\text { Wave: } 52.0 \times 51.0\end{array}$ & & \\
\hline
\end{tabular}

\subsection{Sentinel 2}

Sentinel 2 is an optical imaging mission that utilises a multispectral system for land and coastal region monitoring. The mission consists of a constellation of two satellites: Sentinel 2A and Sentinel 2B (Table 4). The sensors cover 13 spectral bands that range from visible light (blue, green and red), the NIR and SWIR. These bands are utilised for monitoring biophysical characteristics of vegetation, coastal area dynamics, and the detection and classification of land use change.

Table 4 Characteristics of the Sentinel 2 satellites

\begin{tabular}{lllll}
\hline Commission & Spectral bands & Spatial (metres) & Revisit (days) & Radiometric (bits) \\
\hline $2015(2 \mathrm{~A})$ & Coastal aerosol & Coastal aerosol: 60.0 & 5 & 12 \\
$2017(2 \mathrm{~B})$ & Blue & MS: 10.0 & \\
& Green & Rede: 20.0 & & \\
& Red & Water vapour: 60.0 & \\
& NIR & SWIR (cirrus): 60.0 & \\
& Red Edge (4 bands) & SWIR (2 bands): 20.0 & \\
& Water vapour & & \\
& SWIR (cirrus) & & \\
& SWIR (2 bands) & & \\
\hline
\end{tabular}

\section{$4 \quad$ Verification and results}

Sentinel 1 and Sentinel 2 imagery of test site TSFB1 was captured on 14 December 2019. A true colour composite of the Sentinel 2 imagery is presented in Figure 4. The SWIR of Sentinel 2 and the backscatter of Sentinel 1 is presented and contrasted in Figure 5. Note the areas of strong absorption of SWIR and the low SAR backscatter, both of which are indicative of water. Note too, the areas of low absorption of SWIR and low SAR backscatter, which may indicate the presence of very dry surfaces. The final very dry surface probabilistic model is presented in Figure 6.



Figure 4 Illustration of true colour composite (red, green, and blue) of TSFB1 


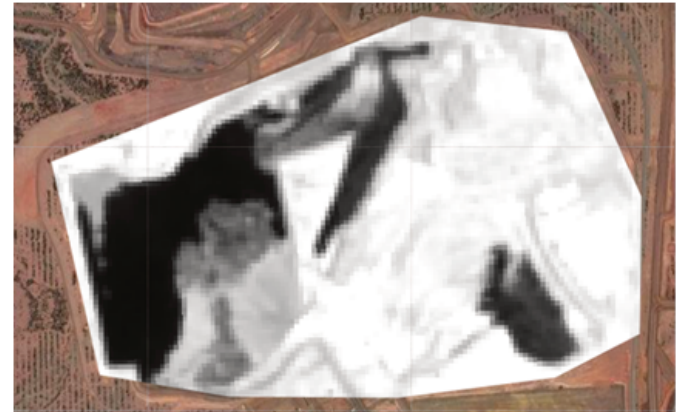

SWIR

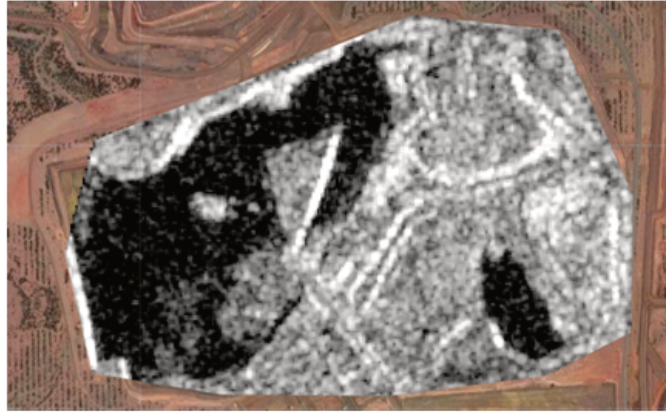

SAR Backscatter

Figure 5 Comparison of SWIR absorption and SAR backscatter for TSFB1

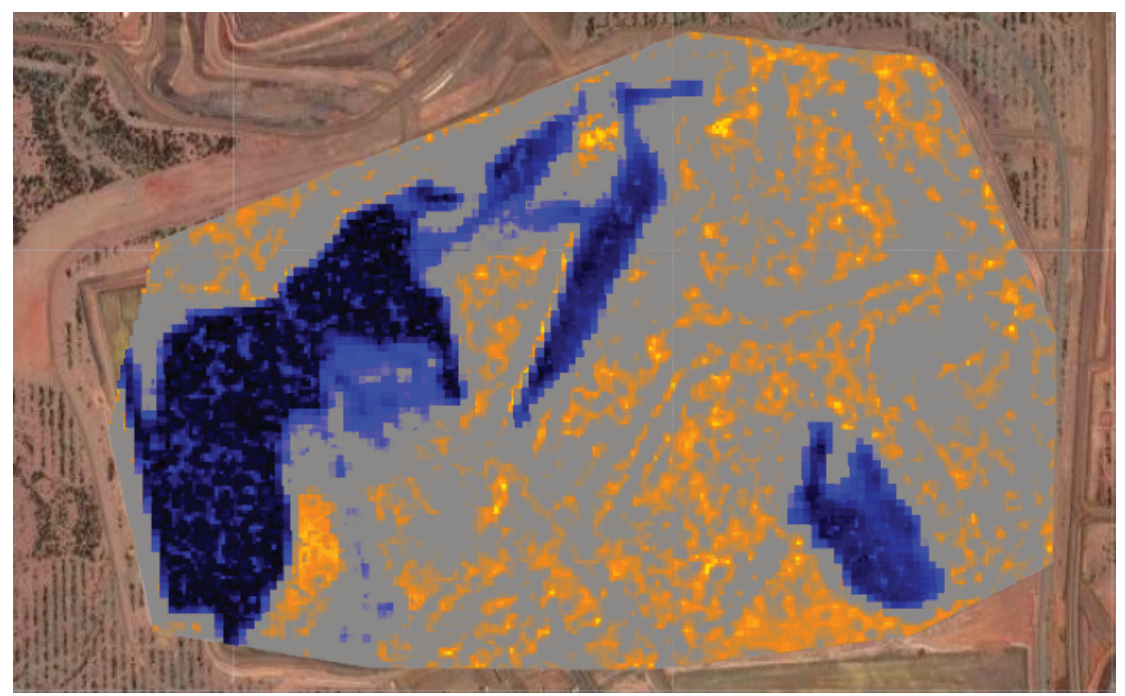

Figure 6 Per-pixel probability of membership to the very dry cluster. Memberships closer to very dry are represented in orange, while memberships far away are represented in blue

\subsection{Time-series demonstration}

A demonstration of the time-series analysis was conducted on another test site; TSFB2. A true colour composite illustration of TSFB2 is presented in Figure 7. A time-series graph of very dry probability between December 2016 and May 2021 at the indicated point in Figure 7 is presented in Figure 8. The highest very dry surface probability at this point occurred on 8 October 2019 (0.914) where tailings was deposited from one spigot location in the southwest corner as other spigots around the facility began to approach their maximum deposition levels causing ponding to occur along the eastern side away from the time-series point. While the lowest occurred on 16 July 2019 (0.069) which coincided with a large pond forming in the area of the time-series point. The probability distributions for these two dates are presented in Figure 9. 


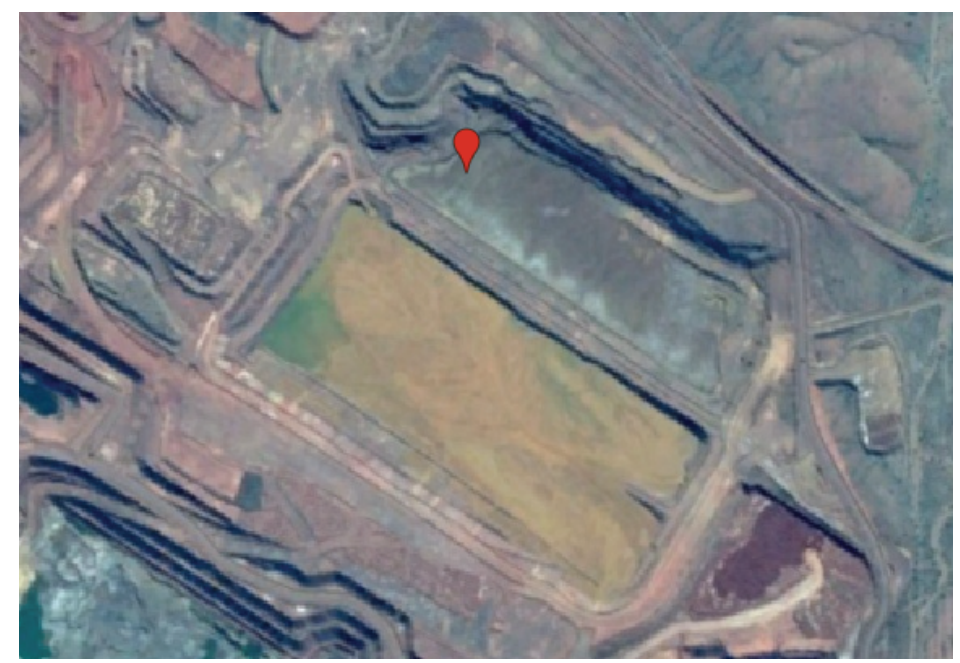

Figure 7 Illustration of TSFB2. Depicted is the point whereby a per-pixel time-series analysis was conducted

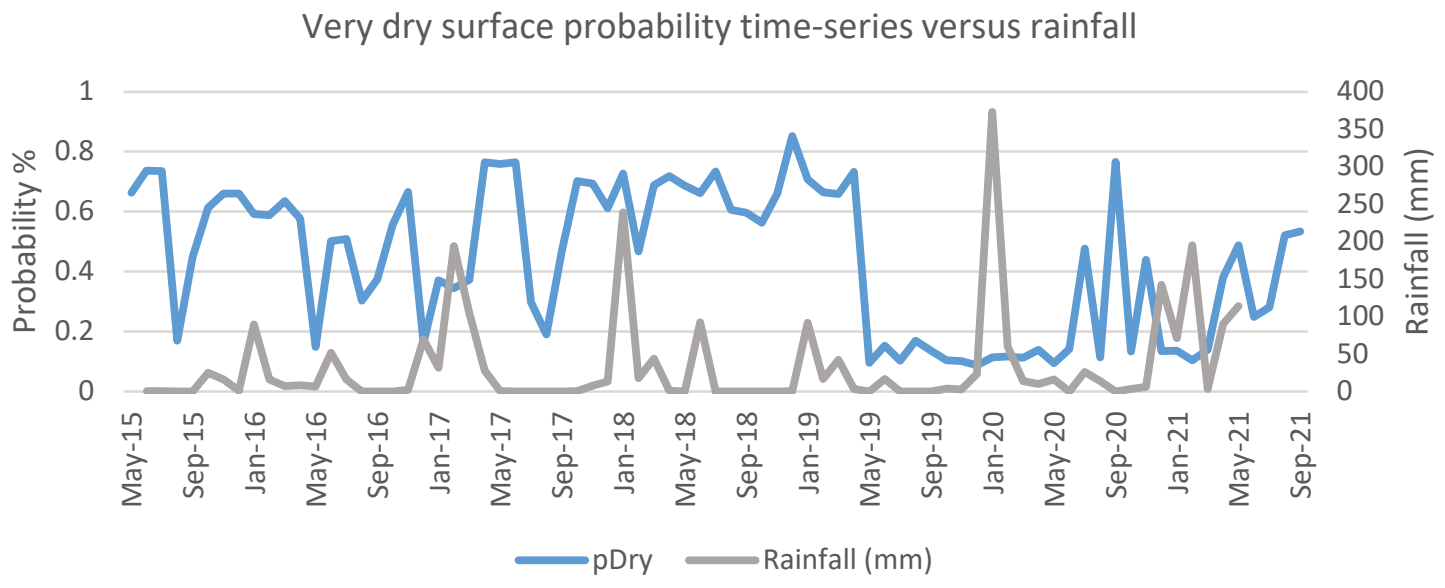

(a)

Very dry surface probability time-series versus tailings deposition

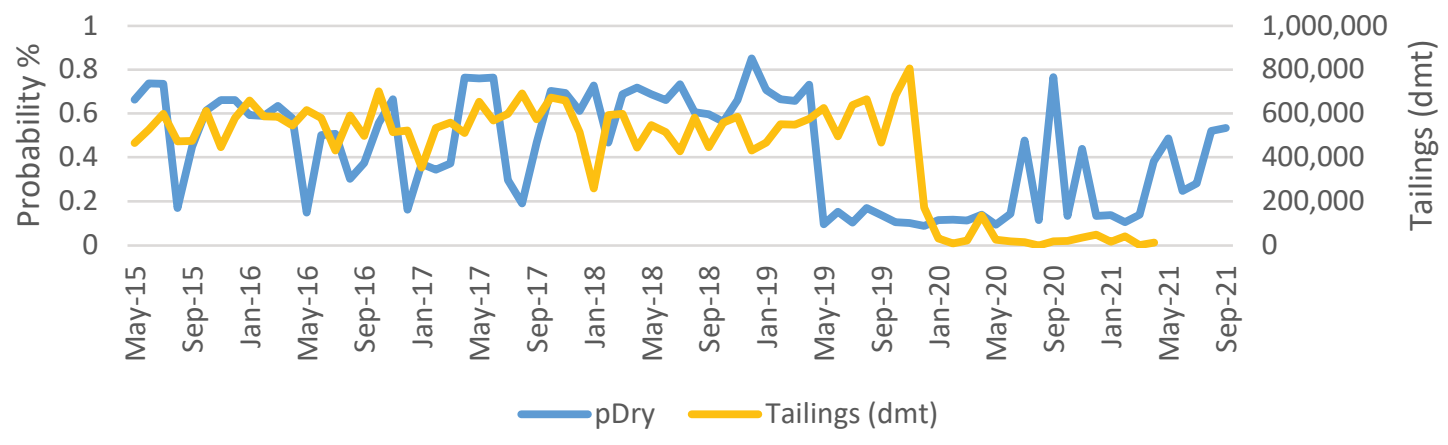

(b)

Figure 8 Time-series graph of very dry surface probability taken at a single point within TSFB2 versus (a) rainfall and (b) tailings deposition 


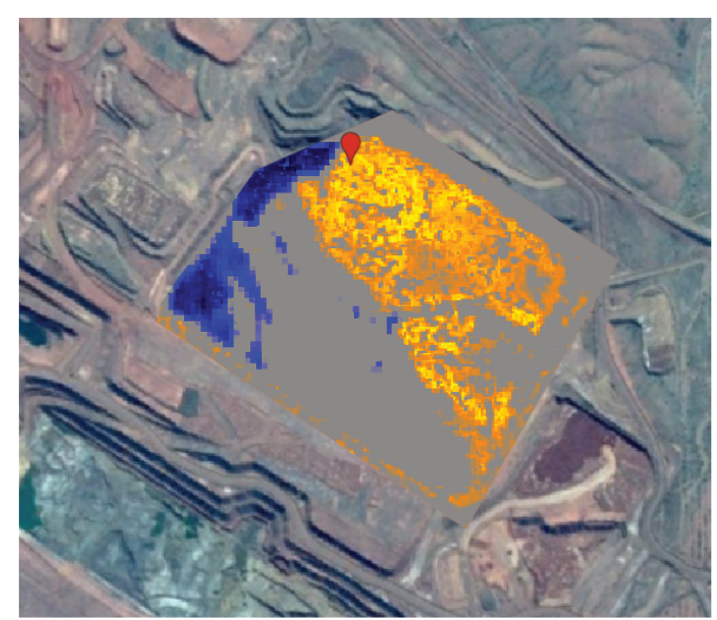

October 82019

(a)

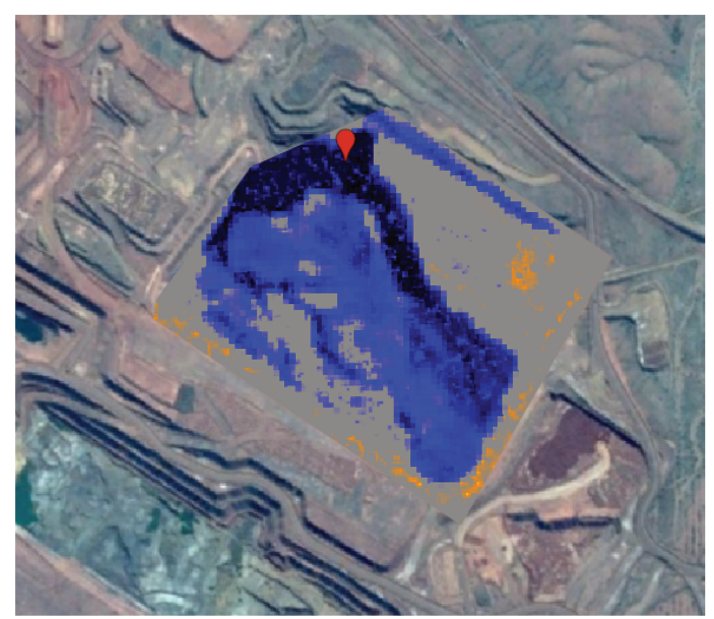

July 162019

(b)

Figure 9 Illustration of the per-pixel distribution of very dry surface probability for the two dates of (a) driest and (b) wettest

\subsection{Comparison against field observations}

The performance of the GEE-based monitoring was reconciled against events recorded by onsite monitoring. An example of an event is the passing of tropical cyclone (TC) Veronica. TC Veronica passed over the project site and TSFA1 between 24 and 25 March 2019. This resulted in $550 \mathrm{~mm}$ of onsite rainfall. The surface of TSFA1 was covered for an estimated four weeks, before drying out significantly by July 2019.

A very dry surface probability time-series was generated for TSFA1 (Figure 10). Noticeable is the large trough of low probability coinciding with the passing of TC Veronica in mid-March 2019. The probability levels within the graph do not return to pre-TC Veronica levels until approximately July 2019. An exploration of the probability imagery over this time period illustrates the effects of inundation followed by progressive drying (Figure 11).

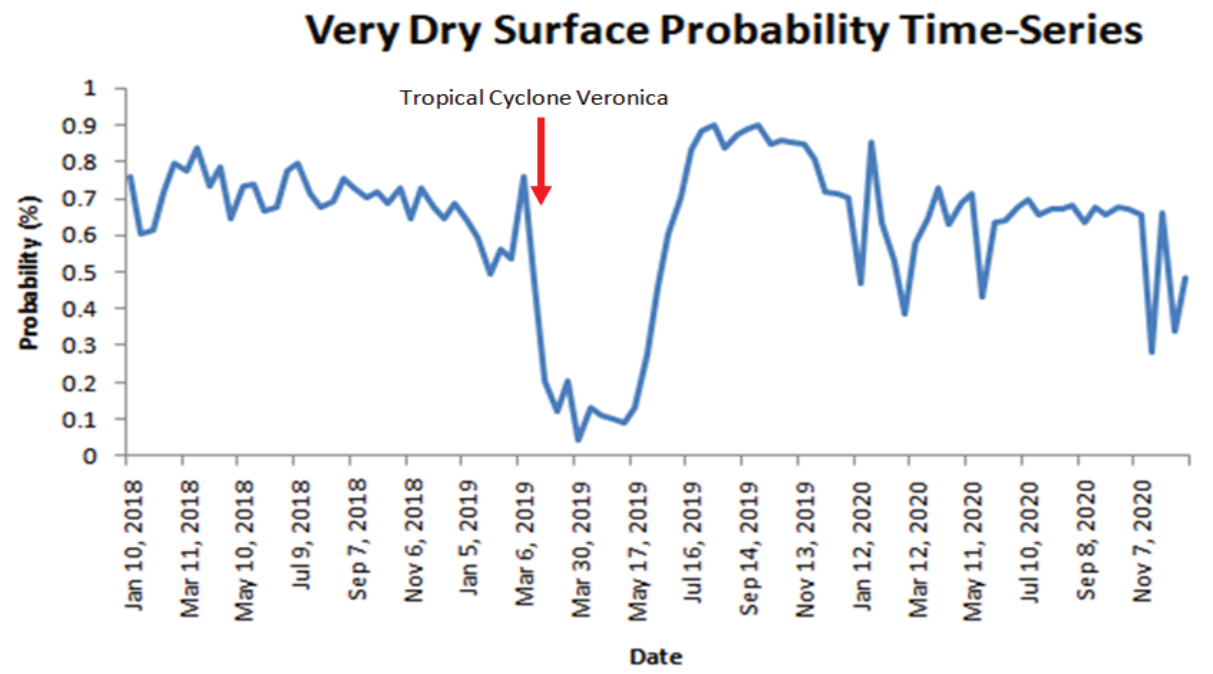

Figure 10 Time-series graph of very dry surface probability taken at a single point within TSF1A 


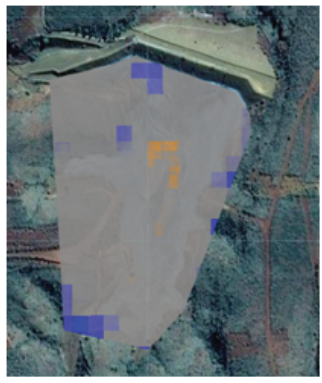

March 6

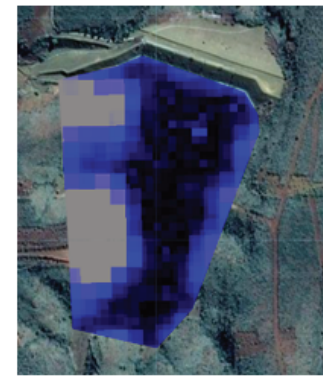

March 30

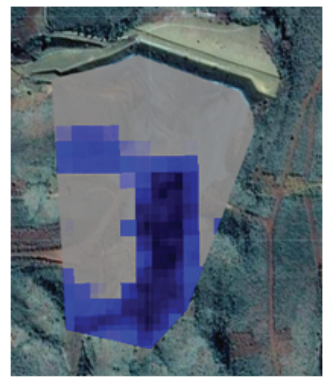

May 29



July 16

Figure 11 Illustration of the per-pixel distribution of very dry surface probability for TSF1A pre and post TC Veronica

\subsection{Anomalous dryness classification}

On 22 November 2018, an on-ground survey observed what would be defined as very dry conditions within the northeast corner of TSF1A. The corresponding GEE analysis of this area at this time, however, reported a relatively moderate probability of water. Furthermore, the GEE predicts an expanding area of water over time in an area that was known to be very dry. Two fundamental possibilities for this confusion are classification uncertainty between the water and very dry clusters, or the potential presence of material with water-like absorption of SWIR.

Additional samples of RS data were extracted from the anomalous area and compared against the original sampling. It was found these predictions lay close to the boundary between dry and water clusters (Figure 12). As the modelling was constrained to only water and very dry clusters, it does not take into account the possibility of a third cluster: dry. It is possible that these samples may belong to this spectrally closer, but unrepresented, dry cluster.

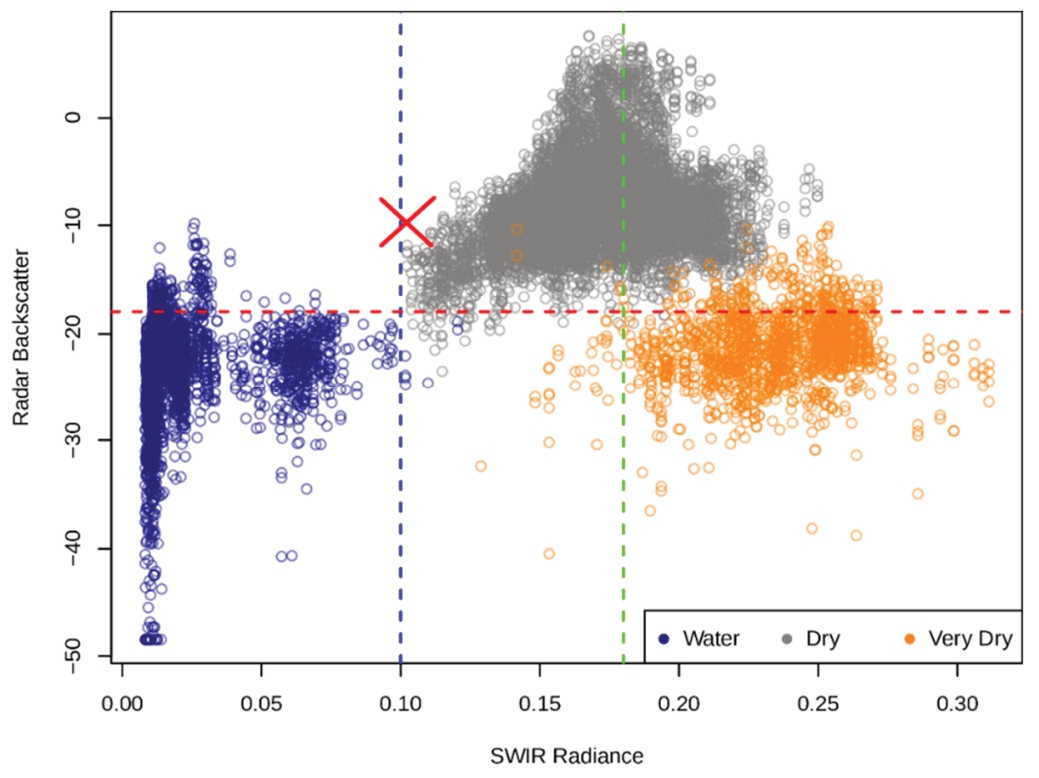

Figure 12 Scatter plot illustrating the relationship between radar and SWIR for three sampled TSF landcover classes: water, dry, and very dry. The red cross marks the location of samples taken from the anomalous area

A second possibility exists whereby the absorption of SWIR is not related to water, but to other materials. The Sentinel 2 SWIR band used in this study has a central wavelength of 2,200 $\mathrm{nm}$. Of the two SWIR bands available from Sentinel 2, this has the wavelength with the greatest value range between wet and dry sandy material found at the study sites (Figure 13). This SWIR wavelength may, however, coincide with absorption characteristics of other materials. 


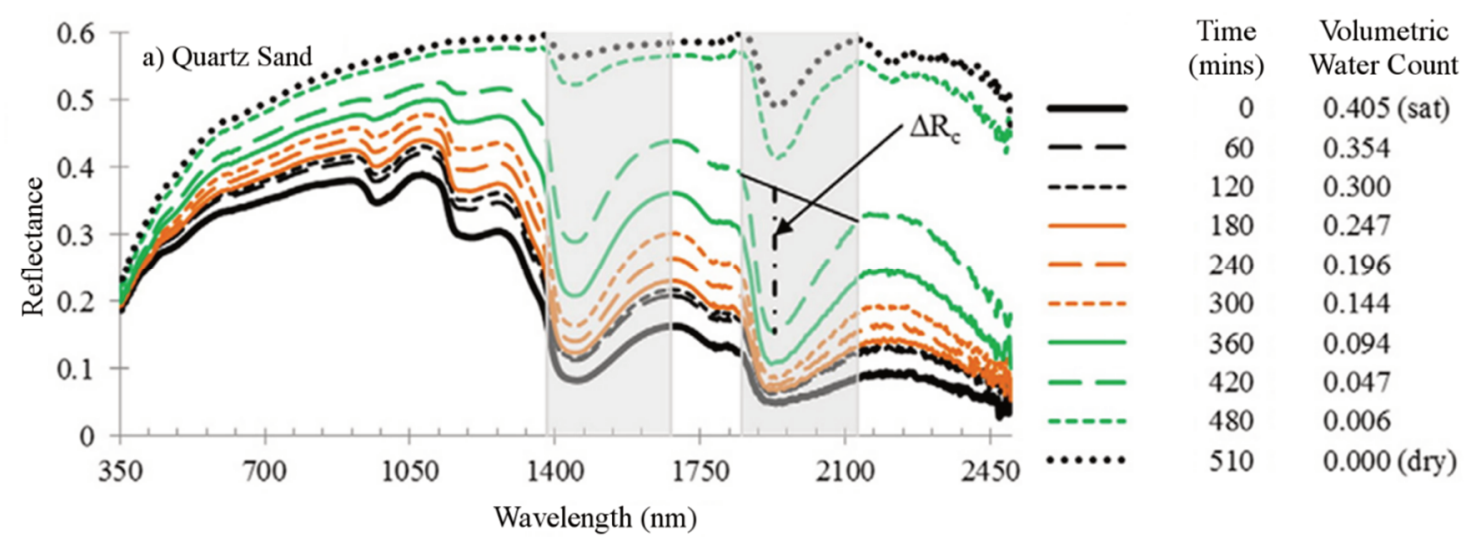

Figure 13 Comparison of the spectral reflectance response of quartz sand as it shifts from a saturated to dry state over time (Tian \& Philpot 2015)

Evidence of SWIR absorption at 2,200 $\mathrm{nm}$ can be found within the literature. Laukamp (2011) discusses examples of 2,200 nm SWIR absorption of members of the di-octahedral phyllosilicate, inosilicate and sulphate mineral groups. The reflectance curves of lead and zinc arsenate minerals, many of which reflect low values at around the 2,200 nm wavelength level, were published by McConachy et al. (2007).

Historical geochemical testing of the tailings deposited into TSF1A identified two predominant minerals that fall into the categories identified by Laukamp (2011): fibroferrite ( 0 to $33 \%$ ) and kaolinite ( 0 to $3 \%$ ). It is, therefore, possible that these minerals have become concentrated within this area. It is not known if this is responsible for the low SWIR reflectance. Given the extended period of no rainfall leading up to the anomalous observation, it is more likely that this absorption is due to the presence of a mineral, rather than water. Although anomalous, the causes of this event and weather data can be used to infer very dry conditions/material presence.

\subsection{Additional benefits}

A number of additional benefits were noted at active TSFs that were outside the original project scope. A false colour composite map of NIR in the green and blue channel, and SWIR in the red channel was developed as shown in Figure 14. NIR in pure water is absorbed at a depth of $12 \mathrm{~cm}$ and SWIR at $1 \mathrm{~mm}$; these depths (ambiguity adjusted for tailings inclusion in the water) allowed the depths of water to be mapped. From field observations and spigotting rotations, it was confirmed that green indicated areas of wet and active tailings deposition with channels and flow paths clearly visible, and that blue indicated ponding of water.

These maps were exported from GEE and polygons assigned to the three colours, red, green, and blue to show the extents of dry tailings, wet tailings and water respectively, within the TSFs (Figure 15). The original assumptions relied upon for the water balance were an assumed area of dry and wet tailings (one third the size of the overall TSF) with the pond area estimated and adjusted from visual observations, quarterly aerial imagery and monthly LiDAR data. Being able to utilise the moisture maps to quantifiably measure the area of these zones showed the pond area was, on average, being overestimated by $37 \%$, the dry beach by $15 \%$, and the wet beach being underestimated by $68 \%$, demonstrating a significant improvement in accuracy of the water balance. The overestimation of the pond volumes alone saw an average reduction of $120,000 \mathrm{~m}^{3}$ of water for TSFB1, and 14,000 $\mathrm{m}^{3}$ for TSFB2.

The ability to map flow paths and pond developments at 11-day intervals also has the added benefit of pond monitoring and control to further mitigate the risks ponding adjacent to embankments and failure on TSFs which are at risk of this. 


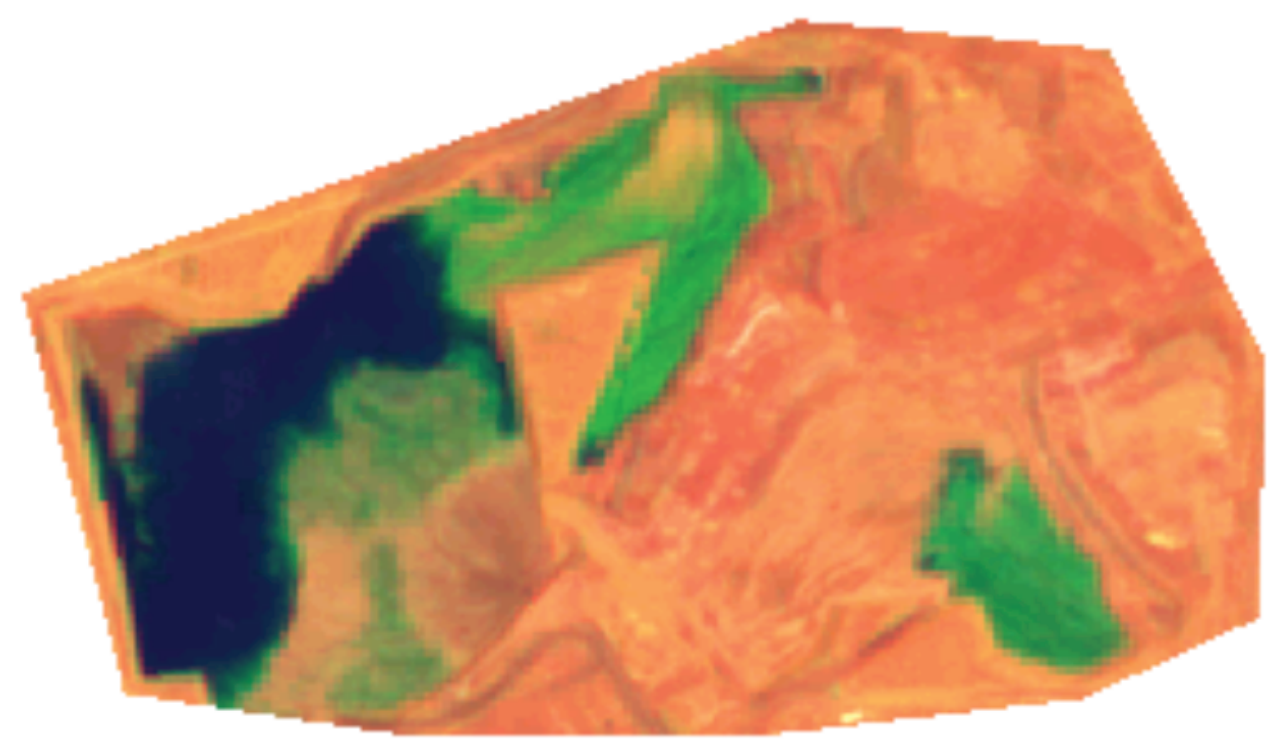

\section{SNG 14 December 2019}

Figure 14 False colour composite using R:G:B = SWIR:NIR:green

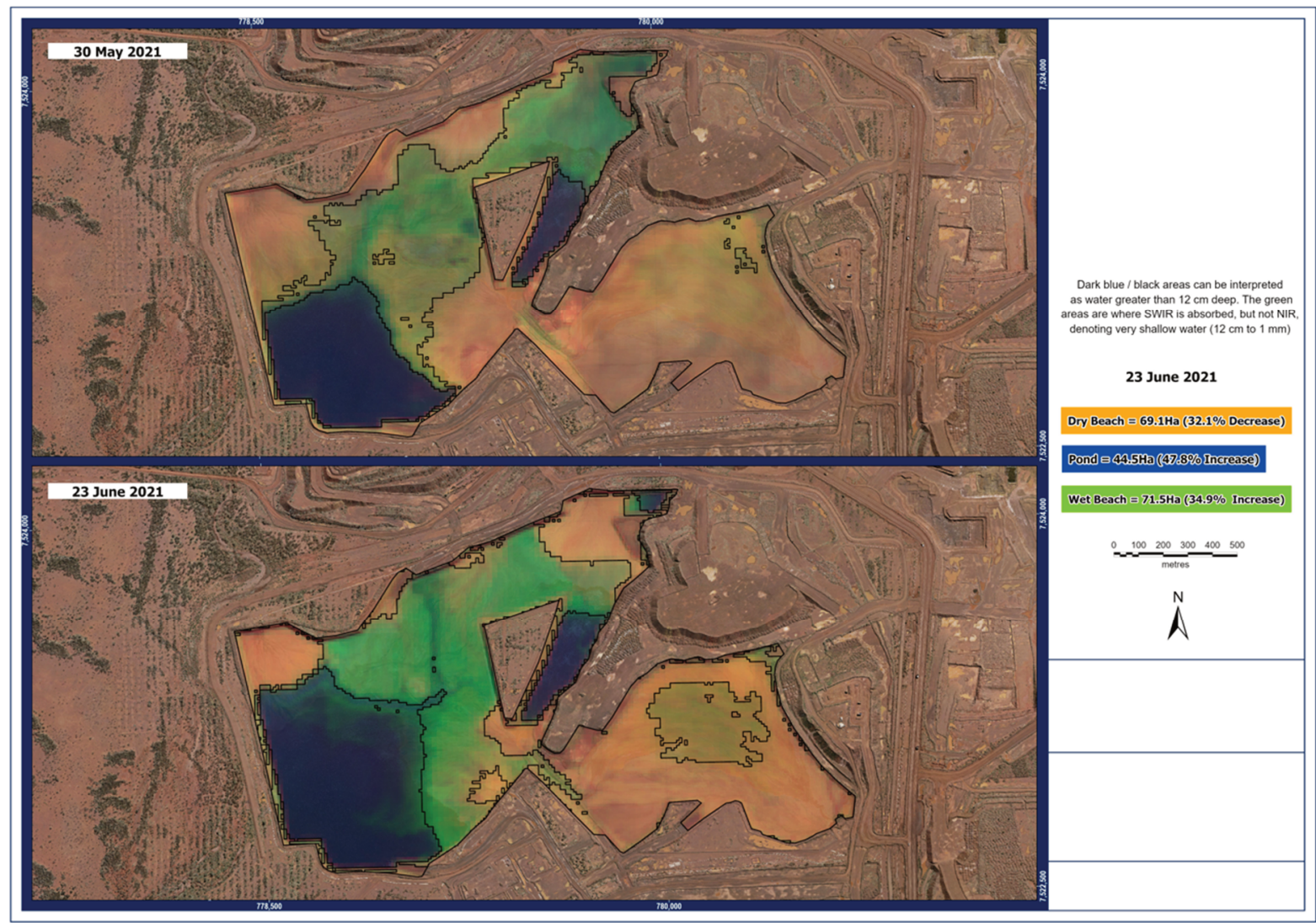

Figure 15 False colour composite using R:G:B = SWIR:NIR:green exported from GEE and areas polygonised based on colour

\section{$5 \quad$ Conclusions and further work}

With the exception of the anomalous result at TSF1A, discussed previously, the fusion of SAR and SWIR imagery has demonstrated, with verification by on-ground observation, that water and very dry material can be distinguished and mapped on TSFs. This distinction in the case of TSF1A allowed for tailings drying 
progression to be monitored onsite and assessed from historical records to the point where it was observed to be very dry, and the feedback correlated to better pinpoint the dusting point based on site observations in November 2019, which was broadly consistent with the values assumed from the backscatter response and SWIR reflectance shown in Figure 3. The relatively large pixel sizes of the satellite imagery ( 20 by $20 \mathrm{~m}$ ) make it difficult to discern the points of very dry and dusting. The engine is therefore currently best used to indicate when tailings is becoming susceptible to dusting based on a very dry feedback, and with further testing and improvement it will likely be able to be used as a predictive tool.

It was noted that dusting points of individual types of tailings will differ and are dependent on the mineralogy and classification properties. The application of this approach therefore needs to be tested and calibrated separately for each TSF. Calibration of the backscatter response and SWIR reflectance to determine the very dry and dusting points will need to be undertaken once TSFA2 is operational. Further lab testing work on samples taken from TSF1A is underway to ascertain the moisture and wind requirements for the tailings at TSF1A to undergo lift-off. This data will then be used in a campaign of moisture testing of the TSF surface at satellite pass-over dates to quantifiably refine the backscatter response and SWIR reflectance points to better understand the very dry and dusting points to inform the application to TFSA2 once constructed.

\section{Acknowledgement}

The authors thank Damien O'Grady, Sam Atkinson and Leroy Du Plessis without whose work this project could not have been completed.

\section{References}

Bousbih, S, Zribi, M, El Hajj, M, Baghdadi, N, Lili-Chabaane, Z, Gao, Q \& Fanise, P 2018, 'Soil moisture and irrigation mapping in a semi-arid region, based on the synergetic use of Sentinel-1 and Sentinel-2 data', Remote Sensing, vol. 10, no. 12, https://doi.org/10.3390/rs10121953

Department of Jobs, Tourism, Science and Innovation 2020, Western Australia Iron Ore Profile August 2020, Perth.

Karvonen, J, Simila \& Makynen, M 2005, 'Open water detection from Baltic Sea ice Radarsat-1 SAR imagery', IEEE Geoscience and Remote Sensing Letters, vol. 2, no. 3, pp. 275-279.

Kok, JF, Parteli, EJR, Michaels, TI \& Karam, DB 2012, 'The physics of wind-blow dust and sand', Reports on Progress in Physics, vol. 75, no. 10.

Laukamp, C 2011, Short Wave Infrared Functional Groups of Rock-forming Minerals, report number EP115222, CSIRO Earth Science and Resource Engineering, Bentley.

Liang, J \& Liu, D 2020, 'A local thresholding approach to flood water delineation using Sentinel-1 SAR imagery', ISPRS Journal of Photogrammetry and Remote Sensing, vol. 159, pp. 53-62.

Lillesand, T, Kiefer, RW \& Chipman, J 2015, Remote Sensing and Image Interpretation, John Wiley \& Sons, Inc., Hoboken.

Martinis, S, Kuenzer, C, Wendleder, A, Huth, J, Twele, A, Roth, A \& Dech, S 2015, 'Comparing four operational SAR-based water and flood detection approaches', International Journal of Remote Sensing, vol. 36, no. 13, pp. 3519-3543.

McConachy, TF, Yang, K, Boni, M \& Evans, NJ 2007, 'Spectral reflectance: preliminary data on a new technique with potential for non-sulphide base metal exploration', Geochemistry: Exploration, Environment, Analysis, vol. 7, no. 2, pp. 139-151.

Tian, J \& Philpot, WD 2015, 'Relationship between surface soil water content, evaporation rate, and water absorption band depths in SWIR reflectance spectra', Remote Sensing of Environment, vol. 169, pp. 280-289. 